\title{
Antioxidant activity in different parts of roselle (Hibiscus sabdariffa L.) extracts and potential exploitation of the seeds.
}

\begin{abstract}
The calyx of the roselle plant has long been recognised as a source of antioxidants. The objective of this study was to evaluate antioxidant activity, free radical-scavenging and total phenolic content in other parts of the roselle plant. Roselle seed extracts were found to have the highest antioxidant activity and strongest radical-scavenging activity of all plants tested. Methanol extracts showed a positive correlation between phenolic content and antioxidant activity, as measured by $\beta$-carotene bleaching assay and DPPH radical-scavenging activity. The antioxidant efficacy of roselle seeds in a whole food system was investigated by testing the effect on lipid oxidation in cooked beef patties stored at $4{ }^{\circ} \mathrm{C}$ for 14 days. Results showed that patties treated with roselle seeds had reduced lipid oxidation compared to patties treated with BHT. This study suggests that roselle seeds have the potential to be used as food antioxidants.
\end{abstract}

Keyword: $\beta$-carotene; Antioxidant; DPPH; Hibiscus sabdariffa L.; Lipid peroxidation; Phenolic; Roselle 